\title{
BEATWAVE ACCELERATION EXPERIMENTS AT UCLA
}

\author{
C. Joshi, C. E. Clayton, W. P. Leemans, K. A. Marsh, \\ M. T. Shu, and R. L. Williams \\ University of California, Los Angeles \\ Los Angeles, CA 90024
}

\section{$\underline{\text { Abstract }}$}

The possibility of using high density plasmas as a medium for a high gradient accelerator is being studied experimentally at UCLA. This proof-of- principle experiment uses a large $\mathrm{CO}_{2}$ laser to excite plasma waves with large accelerating fields and a 1.5 $\mathrm{MeV}$ linac to probe the fields. Preliminary results from optical scattering diagnostics indicate that the length $L$ of the wave times the accelerating gradient $E_{a}$ is so far limited to $E_{2} \leq 3.2 \mathrm{MeV}$ whereas the experiment is designed to achieve $\mathrm{E}_{\mathrm{a}} \mathrm{L} \approx 25 \mathrm{MeV}$.

\section{Introduction}

While the future of high energy physics experiments is determined in the short term by the extrapolation of current accelerator technologies, in the long run it may well be determined by what new technologies can be advanced to reduce the size, complexity, and cost of future accelerators. ${ }^{1}$ Plasma accelerators offer perhaps the highest gradient of all feasible accelerator schemes yet proposed due to the fact that there is no electrical breakdown problem in a plasma and that, for typical achievable plasma densities, the available gradients are in the 10 's to 100 's of $\mathrm{GeV} / \mathrm{m}$. There are two two main approaches to to exciting relativistically-moving waves in a plasma; namely, beatwave excitation ${ }^{2-4}$ and wakefield excitation. 5,6 While this paper deals with a beat-excitation, the insights gained are applicable to all plasma-based accelerator schemes.

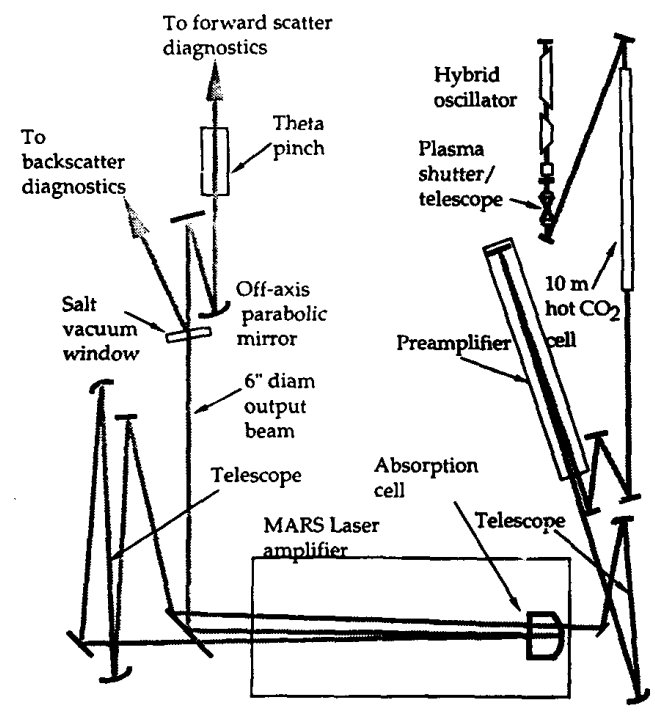

Figure 1: Schematic of the laser system. An absorber cell within the cavity is use to achieve multi-line operation. The beam diameter is about $16 \mathrm{~cm}$ at the output of the final amplifier.
The beatwave is excited when the frequency of the beat of two co- propagating laser beams exactly equals the resonant frequency of the plasma. In our case, the required plasma density, that is, the resonant density, is about $6 \times 10^{16} \mathrm{~cm}^{-3}$. Accelerating fields of more than one $\mathrm{GeV} / \mathrm{m}$ have already been inferred is a similar experiment. ${ }^{7}$ The goal of this experiment is to observe $E_{a}>1 \mathrm{GeV} / \mathrm{m}$ over a length $\mathrm{L}>1 \mathrm{~cm}$ using a low current linac as a test particle injector. In other words, we are looking for $\mathrm{E}_{\mathrm{a}} \mathrm{L}>10$ $\mathrm{MeV}$.

\section{Experimental Setup}

\section{Laser System}

The laser system, shown schematically in Fig. 1, has a front end which produces a $60 \mu \mathrm{J}, 100 \mathrm{psec}$ pulse in two frequencies via optical free induction decay (hybrid oscillator, plasma shutter, and hot $\mathrm{CO}_{2}$ cell). The two wavelengths are 10.27 and $9.56 \mu \mathrm{m}$. The pulse is then amplified to about 15 to $20 \mathrm{~J} /$ /ine in two subsequent amplifiers, the second being pressurized to 2.5 atmospheres. We calculate that the pulse stretches out to about $350 \mathrm{psec}$. Also indicated in Fig. 1 are the paths for collection of forward and backward scattered radiation from the "theta pinch" plasma source.

\section{Electron Injector}

The electron linac used in this experiment is a commercially obtained X-ray source ${ }^{8}$ which has been modified to be an electron machine. Because the experiment is operated in fill gas of roughly one Torr of helium, a vacuum window on the linac was necessary. The window material is $6 \mu \mathrm{m}$ thick Mylar. Upon hitting the Mylar window, the $1 \mu \mathrm{m}$ beam blows up to about $1 \mathrm{~cm}$ at the location of the first solenoidal lens (see Fig. 2). This lens brings the electron beam to a $1 \mu \mathrm{m}$ diam waist and through a hole in the surface of the $\mathrm{CO}_{2}$ laser off-axis paraboloid focusing mirror, as shown in Fig. 2.



Figure 2: Schematic of the electron injector. The $\mathrm{CO}_{2}$ beam enters through the salt window and is focused coaxially with, and to the same spot, as the electron 
A second solenoidal lens focuses the electron beam tightly at the same location as the $\mathrm{CO}_{2}$ laser beam focus, inside the plasma source.

The focusable average beam current from the $9 \mathrm{GHz}$ linac is about $5 \mathrm{~mA}$ with a maximum energy of $1.5 \mathrm{MeV}$. The number of electrons per 20 psec micropulse is about $3 \times 10^{6}$ and, with spot size of about $1 \mathrm{~mm}$, the electron density of the focused beam is about $6 \times 10^{8} \mathrm{~cm}^{-3}$. Synchronization of the linac and laser pulse is left to chance since the $350 \mathrm{psec}$ laser pulse is bound to overlap one of the $9 \mathrm{GHz}$ linac pulses.

\section{$\underline{\text { Diagnostics }}$}

The experiment is diagnosed through optical scattering techniques as well as with test particle injection. The optical techniques consist of spectral analysis of back and forward scattered light. The backscattered light arises from two common instabilities of high intensity light propagation in a plasma. One is Stimulated Brillouin Scattering (SBS) and the other is Stimulated Raman Scattering (SRS). The SBS light signifies the presence of large amplitude ion acoustic wave which can have a detrimental impact on the growth of the beatwave. The SRS light arises from scattering off moderate amplitude, slow phase velocity electron plasma waves. This SRS light is frequency shifted by the plasma frequency (which is simply related to the plasma density). We monitor this frequency shift on every laser shot and thus have an on-line diagniostic of the plasma density at the interaction point.

\section{Electron Detection}

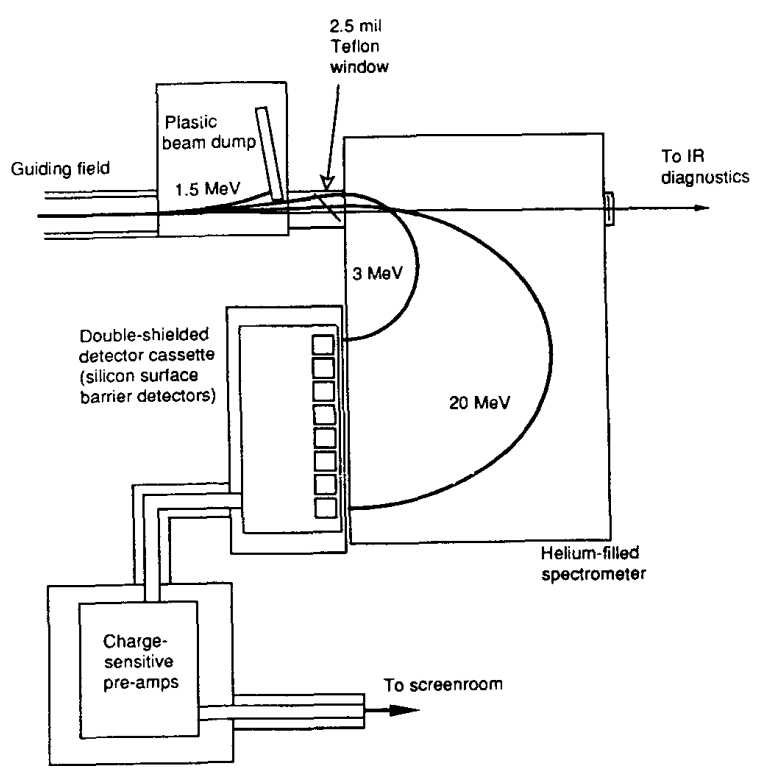

Figure 3: Schematic of the output end of the experiment. An optional beam scraper is used to reduce the noise level on the detectors to below the signal of a single electron.
Another diagnostic is light scattered in the forward direction. This arises when the laser-driven beatwave couples back onto the two laser frequencies to produces sidebands (called Stokes and antiStokes radiation) shifted in frequency by multiples of $\omega_{\mathrm{pe}}$, the plasma frequency. Since these arise as a necessary by-product of the beatwave excitation, they provide a monitor of amplitude and extent of the beatwave.

The arrangement for detecting accelerated electrons is shown in Fig. 3. To keep X-ray noise within the large $180^{\circ}$ spectrometer to a minimum, an optional beam scraper is used, as shown in Fig. 3 , to dump the thousands of micropulses which are not synchronized with the laser pulse. The X-ray noise is distinguishable from an electron signal such that single electrons can be detected.

The plasma source is a classic "theta pinch" operated in a rather non- classical parameter regime. A theta pinch ionizes and compresses a working gas by a fast time-changing magnetic field. Earlier in this experiment we discovered that, when the experiment was timed to operate at the maximum compression of the plasma, which is also near the maximum of the ringing magnetic field, that trapped magnetic fields and other asymmetries in the plasma prevented the electron beam from entering the plasma in a reproducible way. The solution was to find a condition where the resonant density existed at a time when the ringing magnetic field $\mathrm{B}$ was going through zero. This required a fill pressure much higher than one would high densities at maximum compression and allowing the density to drop down to the resonant density as B goes through zero, $4 \mu \mathrm{sec}$ later. That $\mathrm{B}=0$ globally is borne out by the observation of reproducible transmission of electrons in a $200 \mathrm{nsec}$ window about $\mathrm{B}=0$. Unfortunately, the density diagnostic indicates that the plasma is less homogeneous here than at the maximum compression point.

\section{Experimental Results}

Two sets of operating parameters were found to yield the resonant density at $B=0$ in the theta pinch. Condition $I$ is with a fill pressure of about $1.8 \mathrm{~T}$ of helium and a theta pinch capacitor bank charging voltage of $14 \mathrm{kV}$. Condition II is $760 \mathrm{mT}$ of helium and a $24 \mathrm{kV}$ bank voltage.

To establish the resonant density for beatwave excitation for Condition I, we monitored the level and frequency spectrum of SRS scattered light from the plasma. The level of SRS light shifted by the beat frequency, that is, light scattered from the resonant density, is plotted in Fig. 4(a) as a function of initial fill pressure. At the same time we monitored the level of the Stokes signal, which is proportional $\left(\mathrm{E}_{\mathrm{a}} \mathrm{L}\right) 2$. This is plotted in Fig. 4(b). We see a clear resonance in the Stokes signal which correlates with the density measurement. Similar behavior was noted at Condition II.

The variation of the Stokes signal with laser power was also measured. Typical data are shown in Fig. 5(a) for Condition I and in 5(b) for Condition II. Both sets of data show that the amplitude of the beatwave is growing rapidly with laser energy beyond 15-20 $\mathrm{J}$.

Recently, a new IR spectrometer was brought on-line which allows us to monitor the level of "second Stokes" (light scattered in the forward direction with twice the frequency downshift as the Stokes light, i.e. shifted by $2 \omega_{\mathrm{pe}}$ ) simultaneously with the Stokes level. As discussed in the next section, this provides an independent check of the $\mathrm{E}_{\mathrm{a}} \mathrm{L}$ product. For a condition where the Stokes level was $1 / 40$ of the largest level in Fig. 5(b), the second Stokes level was down by a factor of $3.3 \times 10^{3}$. 


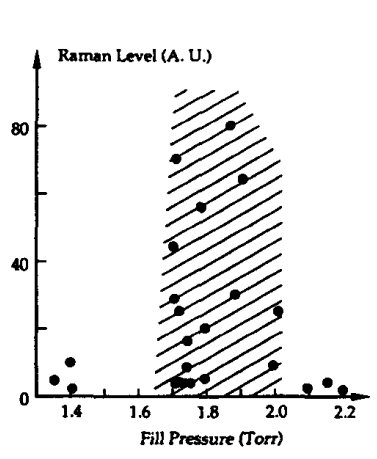

(a)

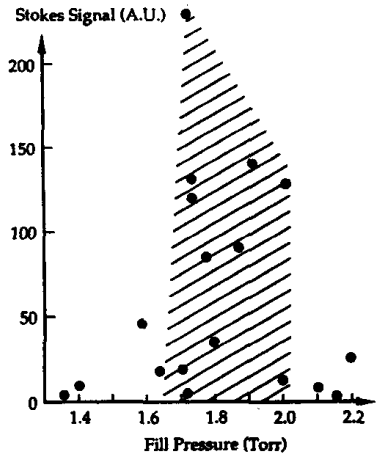

(b)
Figure 4: Levels of Raman scattering from resonanit density (a), and Stokes scattering (b) as a function of fill pressure. The data are from one particular run and no attempt has been made to normalize to variations in incident laser power or line ratio.

\section{Discussion of Results}

The generation of Stokes radiation from the interaction of the beatwave and the laser beams can be viewed as collective scattering of the laser off the organized electrons of the plasma wave. The Stokes power level, $P_{1}$, is related to the incident power, $P_{0}$ by the Bragg scattering relation;

$$
P_{1}=P_{0}\left[\frac{\pi}{2} \frac{\bar{n}}{n_{c}} \frac{L}{\lambda_{0}}\right]^{2}
$$

where $\overline{\mathrm{n}}$ is the electron density perturbation associated with the beatwave, $n_{c}$ is the critical density for laser light of wavelength $\lambda_{0}$, and $L$ is the length over which the wave exists. But $\tilde{n}$ is related to the accelerating field $\mathrm{E}_{\mathrm{a}}$ by Gauss' Law;

$$
\nabla \cdot \mathrm{E}_{\mathrm{a}}=4 \pi \tilde{\mathrm{n}} \mathrm{e}
$$

We can combine Eqs. 1 and 2 to get;

$$
E_{\mathrm{a}} \mathrm{L}=0.079 \sqrt{\mathrm{R}_{0,1}} \mathrm{MeV}
$$

where $R_{0,1}$, is the relative Stokes level in the same units as plotted in Fig. 5. The numerical coefficient takes into account some measured calibration constants as well as some estimated calibration factors. The uncertainty in this coefficient is \pm a factor of 2

Applying Eq. 3 to the peak levels observed in Fig. 5(b), that is $R_{0,1} \approx 1600$, we find that $E_{a} L=3.2 \mathrm{MeV}$. Due to the uncertainty of the numerical coefficient, it is worthwhile to obtain an independent estimate of the field. The relation between the power in the second Stokes, $\mathbf{P}_{2}$, and that in the first Stokes, $\mathbf{P}_{1}$, is completely analogous to Eq. 1 if we replace $L$ by $L / 2$. This is because the scattering beam $P_{1}$ is not constant in space, as was assumed for $P_{0}$. Defining $R_{2,1}=P_{2} / P_{1}$ we can write the Bragg formula as;

$$
\mathrm{E}_{\mathrm{a}} \mathrm{L} \approx 53 \sqrt{\mathrm{R}_{2,1}} \mathrm{MeV}
$$

which gives $E_{\mathrm{a}} \mathrm{L} \approx 0.9 \mathrm{MeV}$ for the shot with $\mathrm{R}_{0,1}$ equal to only 40. This is a promising result since it gives a larger estimate of $E_{g} L$ for $R_{0,1}=40$ than does Eq. 3 by a factor of 1.8 indicating that perhaps the numerical coefficient of Eq. 3 is underestimated by a factor of 1.8. If so, then the largest $\mathrm{E}_{\mathrm{a}} \mathrm{L}$ observed may be more like $6 \mathrm{MeV}$.

The expectations for improving these numbers in the near future is hopeful based on the Stokes data 5(a) which shows the levels growing rapidly with energy. We explain this observation in the following way. At high input energies, the laser amplifier is highly saturated, giving the fastest rising pulses possible, probably under $300 \mathrm{psec}$ rise time. This allows the beatwave to grow to a significant level before the ion acoustic waves associated with SBS have a chance to grow. At low laser powers, SBS, which has a very low threshold, can get ahead of the beatwave and, through a mode coupling process, inhibit the beatwave growth. ${ }^{9}$ We are now in the process of enlarging the laser beam diameter in order to get more laser energy output and push the curve of Fig. 5(b) higher.

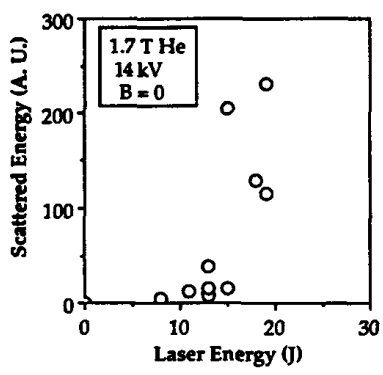

(a)

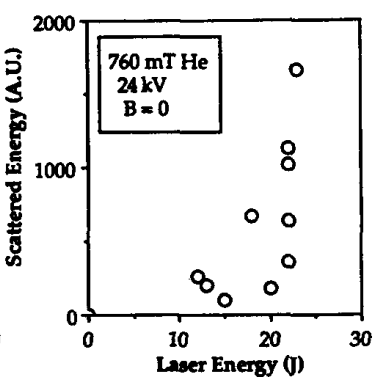

(b)
Figure 5: Levels of Stokes scattering vs laser energy for Condition I (a) and Condition II (b). No attempt was made to normalize data to variations in laser line ratio.

The electron diagnostic has so far not indicated a definite spectrum of accelerated electrons. For this, two obstacles need to be overcome. First, the accelerating field $E_{a}$, here propagating with a Lorentz factor $\gamma_{\phi}=13.5 \mathrm{MeV}$ must be larger than 0.8 $\mathrm{GeV} / \mathrm{m}$ to trap the injected electrons at $\gamma=4$. Second, the number of accelerated electrons must be large enough to make up for a possibly low collection efficiency. In Fig. 6 we show a particle calculation of the number of electrons between 3 and $5 \mathrm{MeV}$ exiting the interaction region within an angular spread of $40 \mathrm{mrad}$ as a function $\mathrm{E}_{\mathrm{a}}$ (note that full scale corresponds to $\mathrm{E}_{\mathrm{a}} \mathrm{L}=3.75$, in line with the experimental estimates). The calculation takes into account the experimental electron beam density and the calculated radial fields of the beatwave. We see that our collection efficiency must be made greater than about $1-2 \%$ in order to see single electrons. We are currently working with the codes TRANSPORT and RAYTRACE to optimize our collection efficiency near $5 \mathrm{MeV}$. 


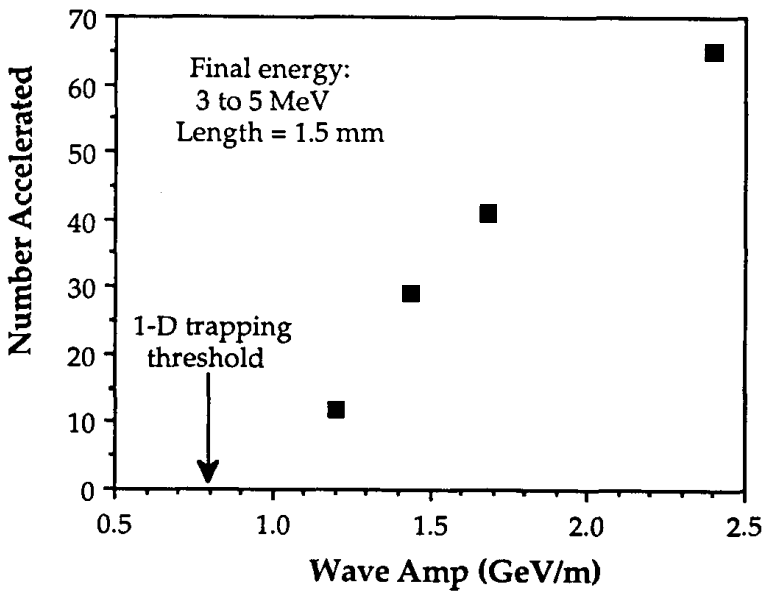

Figure 6: Particle calculation of number of accelerated electrons between 3 and $5 \mathrm{MeV}$ vs the beatwave amplitude. Parameters of the calculation model the experimental parameters.

\section{Current Thrust}

The first round of beat wave acceleration experiments have yielded extremely useful data which can be used to improve the next round of experiments. The three plasma conditions that we have extensively explored using the $\theta$-pinch plasma source in the first series of experiments can be summarized as follows:

Condition

1. Peak Pressure

2. $\mathrm{B}=0,14 \mathrm{kV}$

$1750 \mathrm{mT} \mathrm{He}$

3. $\mathrm{B}=0,24 \mathrm{kV}$

$120 \mathrm{mT} \mathrm{He}$ $750 \mathrm{mT} \mathrm{He}$

\begin{abstract}
Fill Pressure
\end{abstract}
Comments

pinch produces a uniform dense plasma column but $\sim \mathrm{kG}$ trapped field.

pinch acts as preionization source and one must rely on laser to fully ionize: poor homogeneity

pinch over compresses the plasma which expands to give the resonant density at $B=0$, still has tens of guass trapped field. overdamped arc plasma with a coaxial feed such that the return currents are external and symmetric. The peak current is only about $1.2 \mathrm{kA}$ as opposed to $100 \mathrm{kA}$ in a $\theta$-pinch. Also, the time constant of $10 \mu \mathrm{s}$ is longer than $6 \mu \mathrm{s}$ for the $\theta$ pinch. To insure that the preionized plasma forms a nice discharge, the current flows through numerous current limiting resistors such that no localized arcs are formed. The fractional ionization is expected to be tens of percent giving a plasma density of $>2 \times 10^{16} \mathrm{~cm}^{-3}$. We then rely on the laser beam to fully ionize the gas and produce the resonant density.

We are currently upgrading the injector LINAC and the beam-line to improve the injected current by at least a factor 10 . We expect to achieve this by employing independent control of magnetron power, gun voltage and grid voltage necessary to optimize the accelerated current. The circuit arrangement is shown in Fig. 7. The cathode voltage will be controlled by an ultrastable and remote programmable DC high voltage ( $16 \mathrm{kv})$ power supply. A 200 volt battery will keep the grid at cut-off. A small pulser circuit using an avalanche transistor switch and PFN techniques will produce a $5-10 \mathrm{~ns}, 300 \mathrm{~V}$ pulse which will be coupled to the grid via a 1.1 balun type $50 \Omega$ isolation transformer. With these changes we expect $10 \times$ more electrons per microbunch than now available. Further increase in the electron current at the interaction point will be obtained by differentially pumping the electron beam line so that there is no emittance blow-up in going thru a mylar foil which separates the linac from the experiments.

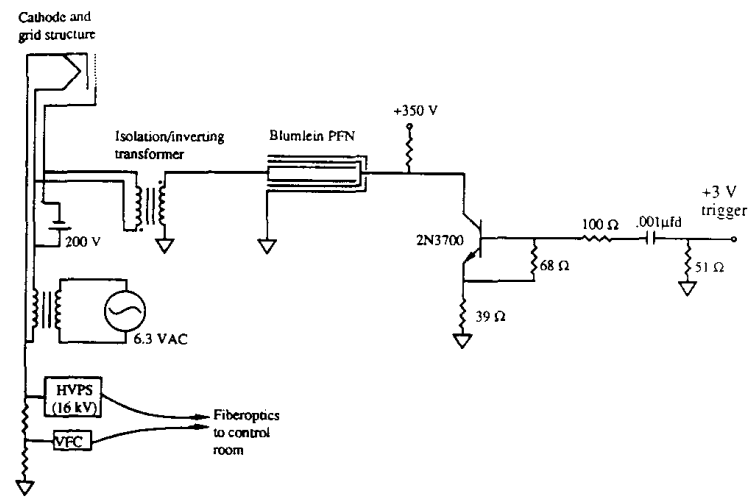

Figure 7: Schematic of the circuitry for gating ( $5 \mathrm{nsec})$ the grid of a DC biased electron gun. The DC bias is isolated from the pulser by a fast $50 \mathrm{~W}$, "balun" type inverting transformer. The high voltage is remoteprogrammable. The transistor switch operates in the avalanche mode. The trigger pulse is derived from the switching pulse for the $\mathrm{CO}_{2}$ laser, thus ensuring synchronization between the laser and electrons. 


\section{Conclusions}

In conclusion, beatwave experiment at UCLA has evolved to a point where we are making quantitative measurements of the wave amplitudes. Scattered light measurements indicate that $\mathrm{E}_{2} \mathrm{~L} \approx 3.2 \mathrm{MeV}$ currently, limited first by laser power (to be scaled up soon) and secondly by plasma homogeneity. Particle calculations indicate that the collection efficiency needs to be $>2 \%$ to begin to see single electrons. With the help of electron- optics codes, we are beginning to optimize the collection efficiency for 5 $\mathrm{MeV}$ electrons.

\section{Acknowledgements}

We would like to thank Drs. W. Mori and T. Katsouleas for many useful discussions. This work supported by DOE contract no. DE-AS03-83-ER40120.

\section{References}

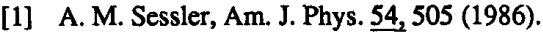

[2] T. Tajima and J. M. Dawson, Phys. Rev. Lett. 43, 267 (1979).

[3] M. Rosenbluth and C. S. Liu, Phys. Rev. Lett. 29, 701, (1972).

[4] C. Joshi et al., Nature $\underline{311_{2}} 525$ (1984)

[5] P. Chen et al., SLAC PUB-3731 (1985) (unpublished). K. L. F. Bane et al., IEEE Tran. Nucl. Sci NS-32, 3524 (1985).

[6] T. Katsouleas, Phys. Rev. A33, 2056, (1986).

[7] C. E. Clayton et al., Phys. Rev. Lett. 54, 2343 (1985).

[8] Schoenburg Radiation Corp., Mountain View, CA.

[9] C. Darrow et al., Phys. Rev. Lett. 56, 2629 (1986). 